\title{
A Nonstandard Finite Difference Scheme for SIS Epidemic Model with Delay: Stability and Bifurcation Analysis
}

\author{
Agus Suryanto \\ Department of Mathematics, Faculty of Sciences, Brawijaya University, Malang, Indonesia \\ Email: suryanto@ub.ac.id
}

Received March 22, 2012; revised April 22, 2012; accepted May 5, 2012

\begin{abstract}
A numerical scheme for a SIS epidemic model with a delay is constructed by applying a nonstandard finite difference (NSFD) method. The dynamics of the obtained discrete system is investigated. First we show that the discrete system has equilibria which are exactly the same as those of continuous model. By studying the distribution of the roots of the characteristics equations related to the linearized system, we can provide the stable regions in the appropriate parameter plane. It is shown that the conditions for those equilibria to be asymptotically stable are consistent with the continuous model for any size of numerical time-step. Furthermore, we also establish the existence of Neimark-Sacker bifurcation (also called Hopf bifurcation for map) which is controlled by the time delay. The analytical results are confirmed by some numerical simulations.
\end{abstract}

Keywords: SIS Epidemic Model with Delay; Stability; Nonstandard Finite Difference Method; Neimark-Sacker (Hopf) Bifurcation

\section{Introduction}

In this paper we reconsider a SIS epidemic model with maturation delay developed in [1]:

$$
\begin{aligned}
\frac{\mathrm{d} s(\xi)}{\mathrm{d} \xi}= & B(n(\xi-\tau)) n(\xi-\tau) e^{-\delta_{1} \tau}-\delta s(\xi) \\
& -\mu \frac{s(\xi) i(\xi)}{n(\xi)}+\gamma i(\xi) \\
\frac{\mathrm{d} i(\xi)}{\mathrm{d} \xi}= & \mu \frac{s(\xi) i(\xi)}{n(\xi)}-(\delta+\varepsilon+\gamma) i(\xi) \\
\frac{\mathrm{d} n(\xi)}{\mathrm{d} \xi}= & B(n(\xi-\tau)) n(\xi-\tau) e^{-\delta_{1} \tau}-\delta n(\xi)-\varepsilon i(\xi) .
\end{aligned}
$$

Here $B(n)$ is a birth rate function. The size of mature population $n(\xi)$ at time $\xi$ is divided into susceptible $s(\xi)$ and infective $i(\xi)$ classes, so that $n(\xi)=s(\xi)+i(\xi) . \delta_{1}, \delta \geq 0$ are the death rates of immature and mature population, respectively. The delay $\tau$ is considered as the maturation time. The parameter $\mu>0, \varepsilon \geq 0$ and $\gamma \geq 0$ are respectively the constant contact rate, the disease induced death rate constant and the recovery constant rate, respectively.

In this paper we consider a special class of Equation (1) by assuming that the death rate in each stage prior to the adult stage and the disease induced death are negligible, i.e. $\delta_{1}=\varepsilon=0$. Using the Rick function as the birth rate function $B(n(\xi))=p e^{-a n(\xi)}$, Equation (1) becomes

$$
\begin{aligned}
\frac{\mathrm{d} s(\xi)}{\mathrm{d} \xi} & =p n(\xi-\tau) e^{-a n(\xi-\tau)}-\delta s(\xi) \\
& -\mu \frac{s(\xi) i(\xi)}{n(\xi)}+\gamma i(\xi) \\
\frac{\mathrm{d} i(\xi)}{\mathrm{d} \xi} & =\mu \frac{s(\xi) i(\xi)}{n(\xi)}-(\delta+\gamma) i(\xi) \\
\frac{\mathrm{d} n(\xi)}{\mathrm{d} \xi} & =p n(\xi-\tau) e^{-a n(\xi-\tau)}-\delta n(\xi) .
\end{aligned}
$$

Note that Equation (2.c) is decoupled and is called the Nicholson's blowflies equation which proposed by [2] The dynamics of Equation (2.c) have been studied by many authors; see e.g. [3,4].

In [1], the basic reproduction number for system (2) has been identified as

$$
R_{0}=\frac{\mu}{\delta+\gamma} .
$$

It has also been shown in [1] that $R_{0}$ determines the existence of equilibria as well as their stability properties. They have shown numerically that if the delay $\tau$ is sufficiently large then the positive solutions of system (2) oscillate about the positive equilibrium. The existence and stability of Hopf bifurcation were established by Wei 
and Zou [5]. In this case, the bifurcation is controlled by parameter $p$. The bifurcation analysis of system (2) using time delay as the control parameter has been done by Chi, Qu and Wei [6].

For practical purposes, we need to do numerical simulations and therefore we have to transform the continuous model (2) into a discrete system. We expect that the dynamical properties of the discrete system are in accordance with its continuous counterpart (2). Kunnawuttipreechachan [7] and the author [8] considered the Euler discretization of system (2) and showed that the discrete system has exactly the same equilibria as those of system (2). Using different method of analysis, Kunnawuttipreechachan [7] and the author [8] derived the sufficient conditions of the numerical step-size for equilibria to be asymptotically stable. It was concluded that the stability conditions of equilibria of the discrete system obtained by the Euler method are consistent with system (2) only if the numerical time-step is relatively small.

To overcome the dependence of stability condition on the time-step size, we will apply a nonstandard finite difference (NSFD) scheme. This method, which is developed by Mickens $[9,10]$, has been applied to various problems; see e.g. [11-16], in which the numerical solutions preserve dynamical properties of the continuous model. We will show that the discrete SIS epidemic model with a delay obtained by the NSFD method maintains the stability properties of equilibria irrespective of the size of numerical time-step. Besides the stability conditions, the existence of bifurcation of the discrete model will also be investigated.

\section{Discrete SIS Epidemic Model with Delay}

Using the fact that $n(\xi)=s(\xi)+i(\xi)$, we will only consider the last two equations of system (2). Using a transformation $I(t)=i(\xi)$ and $N(t)=n(\xi)$ with $\xi=t \tau$, Equation (2.b) and (2.c) can be written as

$$
\begin{gathered}
\frac{\mathrm{d} I(t)}{\mathrm{d} t}=\mu \tau \frac{(N(t)-I(t))}{N(t)} I(t)-\tau(\delta+\gamma) I(t) \\
\frac{\mathrm{d} N(t)}{\mathrm{d} t}=p \tau N(t-1) e^{-a N(t-1)}-\delta \tau N(t) .
\end{gathered}
$$

To discretize system (4) we first consider the step-size of the form $h=1 / k$ where $k$ is a positive integer. Then, applying the forward difference scheme for the derivative and a nonlocal approximation for the right hand sides of system (4) yields a system of difference equations

$$
\begin{aligned}
& \frac{I_{j+1}-I_{j}}{h}=\mu \tau \frac{\left(N_{j+1}-I_{j+1}\right)}{N_{j}} I_{j}-\tau(\delta+\gamma) I_{j+1} \\
& \frac{N_{j+1}-N_{j}}{h}=p \tau N_{j-k} e^{-a N_{j-k}}-\delta \tau N_{j+1},
\end{aligned}
$$

where $I_{j}$ and $N_{j}$ are numerical approximation of $I\left(t_{0}+j h\right)$ and $N\left(t_{0}+j h\right), j=0,1,2, \cdots$, respectively. The numerical scheme (5) is a nonstandard because it uses a nonlocal approximation; see Mickens [9,10]. Here we consider initial conditions

$$
I_{0}=I\left(t_{0}\right) \text { and } N_{m}=\varphi_{m},
$$

where $\varphi_{m}=\varphi\left(t_{m}\right)$, for $m=-k,-k+1, \cdots, 0$. It is easy to show that the implicit scheme (5) can be arranged to get its explicit version, i.e.

$$
\begin{aligned}
I_{j+1} & =\frac{I_{j} N_{j}+h \mu \tau N_{j+1} I_{j}}{N_{j}+\tau h\left(\mu I_{j}+(\delta+\gamma) N_{j}\right)} \\
N_{j+1} & =\frac{N_{j}+p \tau h N_{j-k} e^{-a N_{j-k}}}{1+\tau \delta h} .
\end{aligned}
$$

Direct calculations show that the discrete system (7) has a unique equilibrium: $\left(I_{0}^{*}, N_{0}^{*}\right)=\left(0, \frac{1}{a} \ln \frac{p}{\delta}\right)$ if $R_{0}<1$ and $p>\delta>0$. This equilibrium is called the disease free equilibrium (DFE). On the other hand, if $R_{0}>1$ and $p>\delta>0$ then, in addition to the DFE, there also exists an endemic equilibrium (EE): $\left(I_{e}^{*}, N_{e}^{*}\right)$ where

$$
I_{e}^{*}=\left(1-\frac{1}{R_{0}}\right) N_{e}^{*} \text { and } N_{e}^{*}=N_{0}^{*}=\frac{1}{a} \ln \frac{p}{\delta} .
$$

We observe that those equilibria are exactly the same as those of the continuous system (4); see e.g. [7].

\section{Stability and Neimark-Sacker Bifurcation Analysis}

It is well known that the stability of equilibrium of a dynamical system depends on the distribution of the zeros of its associated characteristics equation. In this section, the distribution of the roots of the characteristics equation will be analyzed using the following results of Zhang, $\mathrm{Zu}$ and Zheng [17].

Theorem 1 (Zhang, Zu and Zheng [17]). Suppose that $\hat{B} \subset R$ is a bounded, closed and connected set; $f(\lambda, \tau)=\lambda^{k}+p_{1}(\tau) \lambda^{k-1}(\tau)+\cdots+p_{k}(\tau)$ is continuous in $(\lambda, \tau) \in C \times \hat{B}$ and $\tau$ is a parameter. Then as $\tau$ varies, the sum of the order of the zeros of $f(\lambda, \tau)$ out of the unit circle:

$$
\{\lambda \in C:|\lambda|>1\}
$$

can change only if a zero appears on or crosses the unit circle.

\subsection{Disease Free Equilibrium}

First we perform a linearization of system (7) about the $\operatorname{DFE}\left(I_{0}^{*}, N_{0}^{*}\right)$ by taking $I_{j}=I_{0}^{*}+u_{j}$ and $N_{j}=N_{0}^{*}+v_{j}$. 
The linearized system around the DFE is given by

$$
\begin{aligned}
& u_{j+1}=\frac{1+\tau h \mu}{1+\tau h(\delta+\gamma)} u_{j} \\
& v_{j+1}=\frac{1}{1+\tau h \delta} v_{j}+\frac{\tau h \delta\left(1-\ln \frac{p}{\delta}\right)}{1+\tau h \delta} v_{j-k} .
\end{aligned}
$$

By introducing new variables

$$
v_{j}=x_{j}^{0}, v_{j-1}=x_{j}^{1}, \cdots, v_{n-k}=x_{j}^{k},
$$

we can rewrite system (8) in the form

$$
\boldsymbol{x}_{j+1}=A \boldsymbol{x}_{j}
$$

where $\boldsymbol{x}_{j}=\left(u_{j}, x_{j}^{0}, x_{j}^{1}, \cdots, x_{j}^{k}\right)^{T}$ and the constant matrix $A$ is defined by

$$
A=\left(\begin{array}{cccccc}
A_{1,1} & 0 & 0 & \cdots & 0 & 0 \\
0 & A_{2,2} & 0 & \cdots & 0 & A_{2, k+2} \\
0 & 1 & 0 & \cdots & 0 & 0 \\
0 & 0 & 1 & \cdots & 0 & 0 \\
\vdots & \vdots & \vdots & \ddots & \vdots & \vdots \\
0 & 0 & 0 & 0 & 1 & 0
\end{array}\right),
$$

with $A_{1,1}=\frac{1+\tau h R_{0}(\delta+\gamma)}{1+\tau h(\delta+\gamma)}, A_{2,2}=\frac{1}{1+\tau h \delta}$, and

$A_{2, k+2}=\frac{\tau h \delta\left(1-\ln \frac{p}{\delta}\right)}{1+\tau h \delta}$. The characteristic equation of matrix $A$ is

$$
\phi_{D F E}(\lambda)=\left(A_{1,1}-\lambda\right) \phi(\lambda)=0
$$

where $\phi(\lambda)=\lambda^{k+1}-A_{2,2} \lambda^{k}-A_{2, k+2}$.

Lemma 1. If $R_{0}<1$ and $p>\delta$ then there exists $a$ $\tau^{*}$ such that all roots of Equation (10) have modulus less than one for $0<\tau<\tau^{*}$.

Proof. The trivial root of Equation (10) is

$$
\lambda_{1}=\frac{1+\tau h R_{0}(\delta+\gamma)}{1+\tau h(\delta+\gamma)} .
$$

It is clear that if $R_{0}<1$ then $\left|\lambda_{1}\right|<1$ for all $\tau>0$. Other roots of Equation (10) are determined by

$$
\phi(\lambda)=\lambda^{k+1}-\frac{1}{1+\tau h \delta} \lambda^{k}-\frac{\tau h \delta\left(1-\ln \frac{p}{\delta}\right)}{1+\tau h \delta}=0 .
$$

when $\tau=0$, Equation (11) becomes $(1-\lambda) \lambda^{k}=0$.

Hence Equation (11), at $\tau=0$, has a root $\lambda=0$ of multiplicity $k$ and a simple root $\lambda=1$. Consider the root $\lambda(\tau)$ such that $|\lambda(0)|=1$. This root depends continuously on $\tau$. Based on Equation (11) we have

$$
\left.\frac{\mathrm{d}|\lambda|^{2}}{\mathrm{~d} \tau}\right|_{\tau=0, \lambda=1}=\lambda \frac{\mathrm{d} \bar{\lambda}}{\mathrm{d} \tau}+\left.\bar{\lambda} \frac{\mathrm{d} \lambda}{\mathrm{d} \tau}\right|_{\tau=0, \lambda=1}=-2 \delta \ln \frac{p}{\delta}<0,
$$

whenever $p>\delta$. Consequently all roots of Equation (10) lie in $|\lambda|<1$ for all sufficiently small $\tau>0$, and the existence of the maximal $\tau^{*}$ follows.

Lemma 2. If $1<\frac{p}{\delta}<e^{2}$ then Equation (11) has no root with modulus one for all $\tau>0$.

Proof. Assume $e^{i \omega^{*}}$ with $0<\omega^{*}<\pi$ be a root of Equation (11) when $\tau=\tau^{*}$. Substituting this root to Equation (11) yields

$$
e^{i(k+1) \omega^{*}}-\frac{e^{i k \omega^{*}}}{1+\tau h \delta}=\frac{\tau h \delta\left(1-\ln \frac{p}{\delta}\right)}{1+\tau h \delta} .
$$

Separating the real and imaginary parts, we have

$$
\begin{aligned}
& \cos (k+1) \omega^{*}-\frac{\cos k \omega^{*}}{1+\tau h \delta}=\frac{\tau h \delta\left(1-\ln \frac{p}{\delta}\right)}{1+\tau h \delta}, \\
& \sin (k+1) \omega^{*}-\frac{\sin k \omega^{*}}{1+\tau h \delta}=0,
\end{aligned}
$$

and therefore we get

$$
\cos \omega^{*}=1+\frac{(\tau \delta h)^{2}\left(2-\ln \frac{p}{\delta}\right) \ln \frac{p}{\delta}}{2(1+\tau \delta h)} .
$$

If $1<\frac{p}{\delta}<e^{2}$ then $\cos \omega^{*}>1$, which yields a contradiction. This completes the proof. $\square$

If $\frac{p}{\delta}>e^{2}$, then the roots $e^{ \pm i \omega^{*}}$ of Equation (11) with modulus one satisfy

$$
\begin{aligned}
& \cos \omega^{*}=1+\frac{(\tau \delta h)^{2}\left(2-\ln \frac{p}{\delta}\right) \ln \frac{p}{\delta}}{2(1-\tau \delta h)} \\
& \tau^{*}=\frac{1}{\delta h}\left(1-\frac{\sin (k+1) \omega^{*}}{\sin k \omega^{*}}\right) \\
& h=\frac{1}{k} .
\end{aligned}
$$

Lemma 3. If $\frac{p}{\delta}>e^{2}$ then the roots of Equation (11) satisfy

$$
d_{h}=\left.\frac{\mathrm{d}|\lambda|^{2}}{\mathrm{~d} \tau}\right|_{\tau=\tau^{*}, \omega=\omega^{*}}>0
$$

where $\tau^{*}$ and $\omega^{*}$ satisfy Equation (15). 
Proof. From Equations (11) and (15) we have

$$
d_{h}=\left[\lambda \frac{\mathrm{d} \bar{\lambda}}{\mathrm{d} \tau}+\bar{\lambda} \frac{\mathrm{d} \lambda}{\mathrm{d} \tau}\right]_{\tau=\tau^{*}, \omega=\omega^{*}}=2 \delta h \frac{\alpha}{\beta},
$$

where

$$
\alpha=\delta^{2} h^{2} \tau(1+2 k+\tau h \delta(k+1))\left(\ln \frac{p}{\delta}-2\right) \ln \frac{p}{\delta}
$$

and

$$
\beta=(1+\tau h \delta)\left(\beta_{1}+\beta_{2}\right)>0,
$$

with

$$
\begin{aligned}
& \beta_{1}=[(1+\tau h \delta)(k+1)-k]^{2} \\
& \beta_{2}=2 k(k+1)(1+\tau h \delta)\left(1-\cos \omega^{*}\right) .
\end{aligned}
$$

It is clear that if $\frac{p}{\delta}>e^{2}$ then $\alpha>0$ and therefore

$$
d_{h}=\left.\frac{\mathrm{d}|z|^{2}}{\mathrm{~d} \tau}\right|_{\tau=\tau^{*}, \omega=\omega^{*}}>0 . \square
$$

Based on Theorem 1 and Lemmas 1-3, we have the following results on stability and bifurcation of system (5) at the DFE.

\section{Theorem 2.}

1) If $R_{0}<1$ and $1<\frac{p}{\delta}<e^{2}$ then the DFE is asymptotically stable for all $\tau>0$.

2) If $R_{0}<1$ and $\frac{p}{\delta}>e^{2}$ then there exists an infinite sequence of time delay parameter $\tau_{0}<\tau_{1}<\cdots<\tau_{j}<\cdots$ such that the DFE is asymptotically stable when $\tau \in\left(0, \tau_{0}\right)$ and unstable when $\tau>\tau_{0}$. Map (7) has a Neimark-Sacker bifurcation at the DFE when $\tau=\tau_{j}$, $j=0,1,2, \cdots$ where $\tau_{j}$ satisfy Equation (15).

\section{Proof.}

1) Let $R_{0}<1$ and $1<\frac{p}{\delta}<e^{2}$ then it is known from Lemmas 1 and 2 that the characteristic Equation (10) has no root with modulus one for all $\tau>0$. Applying Theorem 1, all roots of Equation (10) have modulus less than one for all $\tau>0$. Thus, conclusion (a) follows.

2) Let $R_{0}<1$ and $\frac{p}{\delta}>e^{2}$. It is known from Lemma 1 that if $R_{0}<1$ then one of roots of characteristics Equation (10) has modulus less than one for all $\tau>0$. Other roots of Equation (10) satisfy Equation (11). From Lemmas 1 and 3 we know that all roots of Equation (11) have modulus less than one when $\tau \in\left(0, \tau_{0}\right)$, and Equation (11) has at least a couple of roots with modulus greater than one when $\tau>\tau_{0}$. Hence conclusion (b) fol- lows.

\subsection{Endemic Equilibrium}

The linearization of system (7) around the EE $\left(I_{e}^{*}, N_{e}^{*}\right)$ is performed by substituting $I_{j}=I_{e}^{*}+u_{j}$ and $N_{j}=N_{e}^{*}+v_{j}$ into system (7) to get

$$
\begin{aligned}
& u_{j+1}=B_{1,1} u_{j}+B_{1,2} v_{j}+B_{1, k+2} v_{j-k} \\
& v_{j+1}=B_{2,2} v_{j}+B_{2, k+2} v_{j-k},
\end{aligned}
$$

where

$$
\begin{aligned}
& B_{1,1}=\frac{1+\tau h(\delta+\gamma)}{1+\tau h R_{0}(\delta+\gamma)}, \\
& B_{2,2}=\frac{1}{1+\tau h \delta}, \\
& B_{1,2}=\frac{\tau h(1-\mu+\delta+\gamma)(\tau h \delta(\delta+\gamma)+\delta+\gamma-\mu)}{(1+\tau \mu h)(1+\tau h \delta) \mu}, \\
& B_{1, k+2}=\frac{\tau^{2} h^{2} \delta\left(1-\ln \frac{p}{\delta}\right)(\mu-\delta-\gamma)}{(1+\tau \mu h)(1+\tau h \delta)}
\end{aligned}
$$

and

$$
B_{2, k+2}=\frac{\tau h \delta\left(1-\ln \frac{p}{\delta}\right)}{1+\tau h \delta} .
$$

Using the same transformation as in the DFE, i.e. $v_{j}=x_{j}^{0}, v_{j-1}=x_{j}^{1}, \cdots, v_{n-k}=x_{j}^{k}$, system (16) can be written as

$$
\boldsymbol{x}_{j+1}=B \boldsymbol{x}_{j}
$$

where $\boldsymbol{x}_{j}=\left(u_{j}, x_{j}^{0}, x_{j}^{1}, \cdots, x_{j}^{k}\right)^{T}$. The constant matrix $\boldsymbol{B}$ in Equation (17) is

$$
\boldsymbol{B}=\left(\begin{array}{cccccc}
B_{1,1} & B_{1,2} & 0 & \cdots & 0 & B_{1, k+2} \\
0 & B_{2,2} & 0 & \cdots & 0 & B_{2, k+2} \\
0 & 1 & 0 & \cdots & 0 & 0 \\
0 & 0 & 1 & \cdots & 0 & 0 \\
\vdots & \vdots & \vdots & \ddots & \vdots & \vdots \\
0 & 0 & 0 & 0 & 1 & 0
\end{array}\right) .
$$

It is easy to show that the characteristic equation of matrix $\boldsymbol{B}$ is

$$
\phi_{E E}(\lambda)=\left(B_{1,1}-\lambda\right) \phi(\lambda)=0
$$

where $\phi(\lambda)=\lambda^{k+1}-B_{2,2} \lambda^{k}-B_{2, k+2}$. Clearly this characteristic equation has a trivial root

$$
\lambda_{1}=B_{1,1}=\frac{1+\tau h(\delta+\gamma)}{1+\tau h R_{0}(\delta+\gamma)}
$$


and other roots are determined by $\phi(\lambda)=0$. Direct calculations show that if $R_{0}>1$ then $0<\lambda_{1}<1$ for all $\tau>0$. Since $B_{2,2}=A_{2,2}$ and $B_{2, k+2}=A_{2, k+2}, \phi(\lambda)$ is exactly the same as in Equation (11) and therefore the distribution of its roots is exactly the same as described by Lemmas 1-3. Hence we directly have the following results.

Lemma 4. If $R_{0}>1$ and $p>\delta$ then there exists $a$ $\tau^{*}$ such that all roots of Equation (18) have modulus less than one for $0<\tau<\tau^{*}$.

Lemma 5. If $R_{0}>1$ and $1<\frac{p}{\delta}<e^{2}$ then Equation (18) has no root with modulus one for all $\tau>0$.

Lemma 6. If $R_{0}>1$ and $\frac{p}{\delta}>e^{2}$ then the modulus of trivial root of Equation (18), i.e. $\lambda_{1}=\frac{1+\tau h(\delta+\gamma)}{1+\tau h R_{0}(\delta+\gamma)}$, is less than one for all $\tau>0$ and other roots satisfy

$$
d_{h}=\left.\frac{\mathrm{d}|\lambda|^{2}}{\mathrm{~d} \tau}\right|_{\tau=\tau^{*}, \omega=\omega^{*}}>0
$$

where $\tau^{*}$ and $\omega^{*}$ satisfy Equation (15).

Theorem 3.

1) If $R_{0}>1$ and $1<\frac{p}{\delta}<e^{2}$ then the EE is asymptotically stable for all $\tau>0$.

2) If $R_{0}>1$ and $\frac{p}{\delta}>e^{2}$ then there exists an infinite sequence of time delay parameter $\tau_{0}<\tau_{1}<\cdots<\tau_{j}<\cdots$ such that the EE is asymptotically stable when $\tau \in\left(0, \tau_{0}\right)$ and unstable when $\tau>\tau_{0}$. System (7) has a Neimark-Sacker bifurcation at the EE when $\tau=\tau_{j}$, $j=0,1,2, \cdots$ where $\tau_{j}$ satisfy Equation (15).

Theorems 2.1 and 3.1 give the sufficient conditions for DFE and EE to be asymptotically stable, respectively. Unlike the discrete system obtained by the Euler method where the stability conditions for equilibria depend on the size of time-step $h$, the proposed discrete system (7) has stability conditions which are consistent with the stability conditions for equilibria of continuous system (4) for any size of time step $h$; see [7] for the stability properties of the continuous system. In addition, Theorems 2.2 and 3.2 show the existence of bifurcation controlled by the time delay $\tau$.

\section{Numerical Simulations}

To confirm our previous theoretical analysis, in this section we present some numerical simulations using nonstandard finite difference scheme (7) with time - step $h=$ 0.1 (or $k=10$ ). For the simulations we adopt parameters used by [7], i.e. $\delta=1.1, a=2.0$ and $\gamma=1.0$. For the simulations of DFE and EE scenarios we use $\mu=2.0$ and $\mu=5.0$, respectively.

\subsection{Disease Free Equilibrium $\left(R_{0}<1\right)$}

For simulations of the DFE scenario we use a constant contact rate $\mu=2.0$ and vary the value of $p$. Using this constant contact rate we have that $R_{0}=0.9524<1$. First we choose $p=e^{2}$ and therefore the conditions for the DFE to be stable are satisfied, i.e. $1<p / \delta<e^{2}$. Figure 1 shows the numerical solutions using $\tau=1.0$ and $\tau=4.0$. It is indicated that the numerical solutions are convergent to the DFE $(0.0,0.9523)$ for any $\tau>0$. On the other hand, if we take $p=15$ (i.e., $p / \delta>e^{2}$ ) and other parameters are the same as used in Figure 1 then according to the previous analysis the DFE is unstable and the system undergoes a periodic solution of bifurcation. In this case the bifurcation point is $\tau^{*}=1.6601$. Such behavior is shown in Figure 2. Indeed, if $\tau=1.60<\tau^{*}$ then the numerical solution converges to the DFE $(0.0,1.3064)$. On the contrary, the numerical solution, especially $N(t)$, changes its stability from asymptotic stable to periodic behavior when $\tau=1.70>\tau^{*}$.

\subsection{Endemic Equilibrium $\left(R_{0}>1\right)$}

For the EE scenario we use $\mu=5.0$ such that $R_{0}=$ $2.3810>1$. Figures 3(a) and (b) show the numerical solutions for $p=e^{2}, \quad \delta=1.1$ (i.e $p / \delta<e^{2}$ ), using $\tau=1.0$ and $\tau=4.0$, respectively. These results indicate that if $R_{0}>1$ then the $\operatorname{EE~}(0.5524,0.9523)$ is asymptotically stable irrespective of $\tau$.

Next we take the same parameters as used in Figure 3 but with $p=15$, i.e. $p / \delta>e^{2}$. Theorem 3 predicts that a Neimark-Sacker bifurcation will occur. From Equation (15) we find that the critical delay (bifurcation point) is
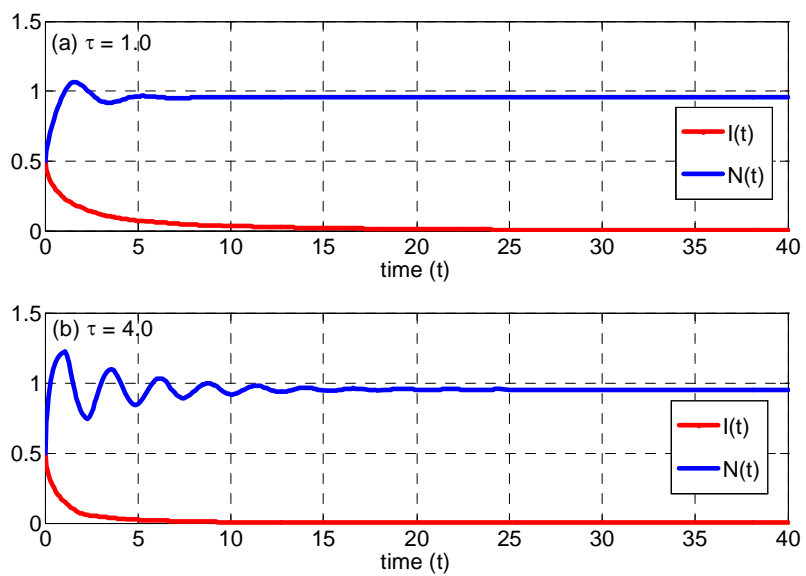

Figure 1. The numerical solutions of the discrete SIS model with a delay for the DFE scenario $\left(R_{0}<1\right)$ where $1<p / \delta<e^{2}$ and (a) $\tau=1.0$ and (b) $\tau=4.0$. 

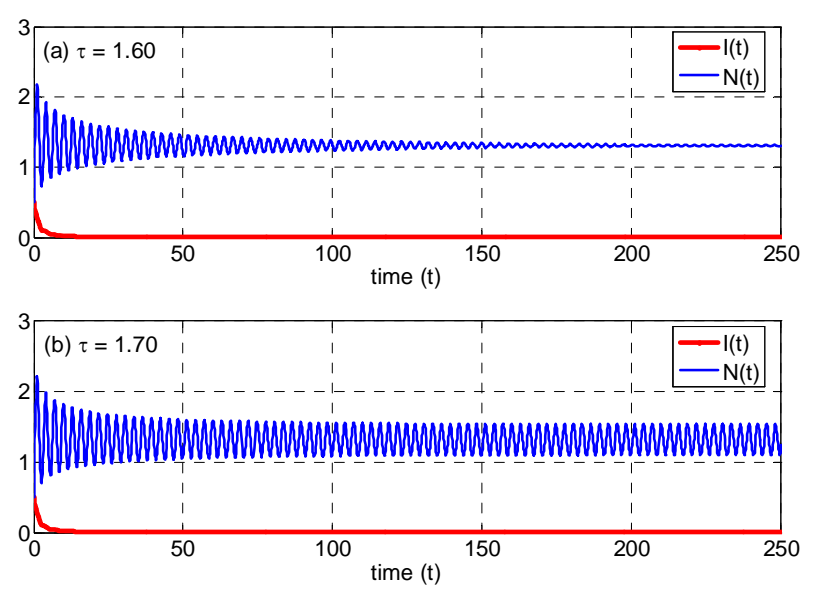

Figure 2. The numerical solutions of the discrete SIS model with a delay for the DFE scenario with $p / \delta>e^{2}, R_{0}<1$ using (a) $\tau=1.60$ and (b) $\tau=1.70$.
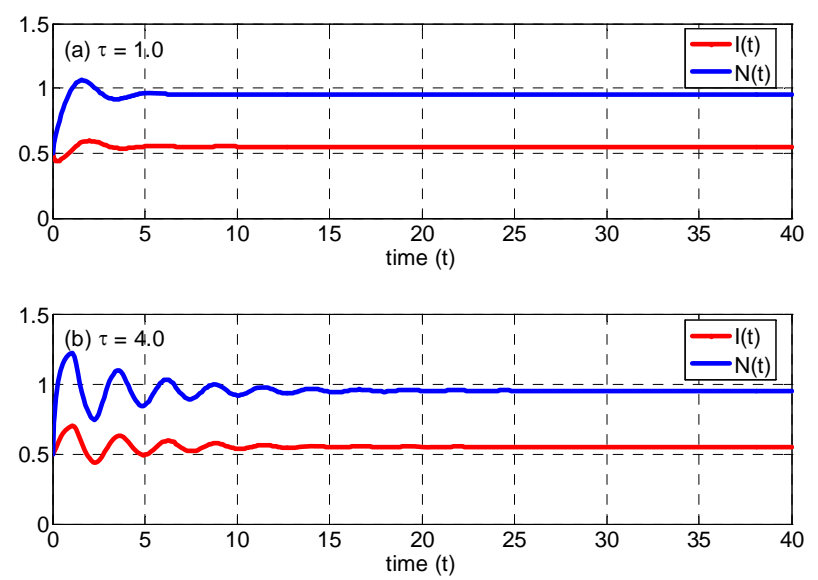

Figure 3. The numerical solutions of the discrete SIS model with a delay for the EE scenario with $1<p / \delta<e^{2}$ and $R_{0}>1$ using (a) $\tau=1.0$ and (b) $\tau=4.0$.

$\tau^{*}=1.6601$. This prediction is confirmed by our numerical solutions; see Figure 4. It is clearly seen that if we change the time delay from $\tau=1.60<\tau^{*}$ to $\tau=1.70>\tau^{*}$, then the behavior of numerical solutions changes from a solution converging to the EE $(0.7577,1.3064)$ to a solution which oscillates periodically about the EE.

Finally we compare the numerical solutions obtained by Euler method with those obtained by our NSFD method. We found that Euler method with a relatively big numerical time-step $(h)$ may produce unrealistic negative and unbounded solutions. However, using the same or even bigger numerical time-step, NSFD method always gives positive and bounded solution. Depending on the parameters used in the simulations, the solution will be periodic or convergent to a correct equilibrium point. For example, in Figure 5 we plot the results of Euler method and NSFD method for the EE scenario using $h=0.25$. It is seen from Figure 5(a) that the
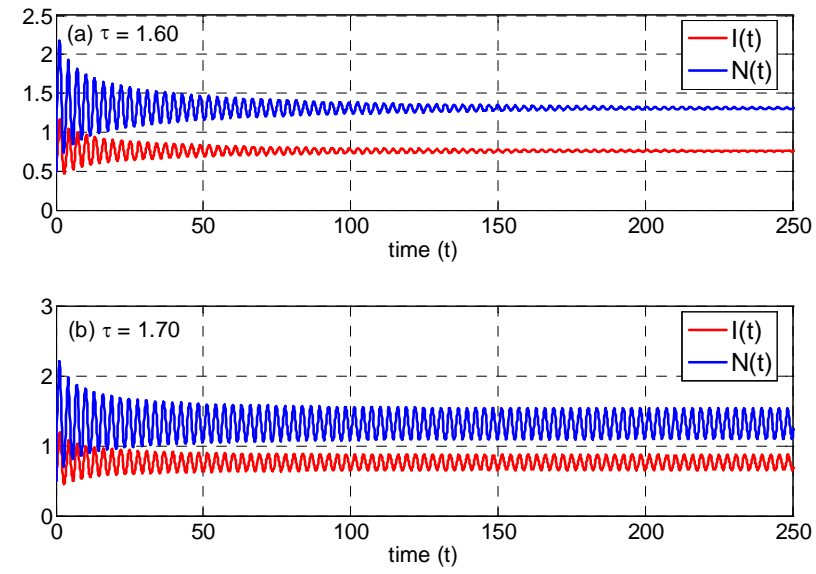

Figure 4. The numerical solutions of the discrete SIS model with a delay for the EE scenario with $p / \delta>e^{2}, R_{0}>1$ using (a) $\tau=1.60$ and (b) $\tau=1.70$.
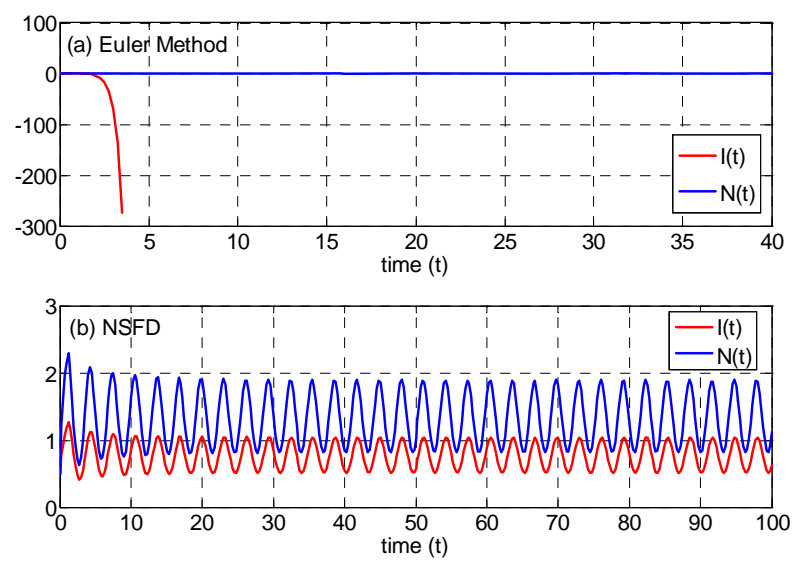

Figure 5. The numerical solutions for the EE scenario with $p / \delta>e^{2}, R_{0}>1, h=0.25(k=4)$ and $\tau=2$ obtained by (a) Euler method and (b) NSFD method. Note that the result of Euler method is plotted in logarithmic scaled.

number of infectives tends to negative infinity (notice that the graph is shown in logarithmic scaled). Using the same value of $h$ the solution of NSFD method oscillates about the EE; see Figure 5(b).

\section{Conclusion}

In this paper we have introduced a discrete delayed SIS epidemic model obtained by a nonstandard finite difference method. Unlike the Euler method, the proposed scheme reproduces exactly the same equilibria as well as their stability conditions as those of continuous model, i.e. if $1<p / \delta<e^{2}$ then the delay does not affect the stability of the equilibria. However, in the case of $p / \delta>e^{2}$, the stability of equilibria changes when the delay passes a critical value. Here the discrete SIS model with a delay has periodic solutions when the stability is lost. In other words, a Neimark-Sacker bifurcation occurs. 
It is also shown that our numerical simulations have confirmed our analytical findings.

\section{Acknowledgements}

This research is supported by Direktorat Penelitian dan Pengabdian kepada Masyarakat, Direktorat Jenderal Pendidikan Tinggi Indonesia (Penelitian Unggulan Perguruan Tinggi-Fundamental) via DIPA Brawijaya University No. 0636/023-04.2.16/15/2012. The author would like to thank the anonymous reviewers for their valuable comments and suggestions to improve this article.

\section{REFERENCES}

[1] K. L. Cooke, P. V. D. Driessche and X. Zou, "Interaction of Maturation Delay and Nonlinear Birth in Population and Epidemic Model," Journal of Mathematical Biology, Vol. 39, No. 4, 1999, pp. 332-352. doi: $10.1007 / \mathrm{s} 002850050194$

[2] W. S. C. Gurney, S. P. Blythe and R. M. Nisbet, "Nicholson's Blowflies (Revisited)," Nature, Vol. 287, No. 5777, 1980, pp. 17-21. doi:10.1038/287017a0

[3] J. Wei and M. Y. Li, "Hopf Bifurcation Analysis in a Delayed Nicholson Blowflies Equation," Nonlinear Analysis, Vol. 60, No. 7, 2005, pp. 1351-1367. doi:10.1016/j.na.2003.04.002

[4] X. Ding and W. Li, "Stability and Bifurcation of Numerical Discretization Nicholson Blowflies Equation with Delay," Discrete Dynamics in Nature and Society, Vol. 2006, 2006, pp. 1-12.

http://www.hindawi.com/journals/ddns/2007/092959/ref/

[5] J. Wei and X. Zou, "Bifurcation Analysis of a Population Model and the Resulting SIS Epidemic Model with Delay," Journal of Computational and Applied Mathematics, Vol. 197, No. 1, 2006, pp. 169-187. doi:10.1016/j.cam.2005.10.037

[6] Q. Chi, Y. Qu and J. Wei, "Bifurcation Analysis of an Epidemic Model with Delay as the Control Variable," Applied Mathematics E-Notes, Vol. 9, 2009, pp. 307-319. http://www.math.nthu.edu.tw/ amen/2009/091020-2.pdf

[7] E. Kunnawuttipreechachan, "Stability of a Numerical Discretization Scheme for the SIS Epidemic Model with a Delay," Proceedings of the World Congress on Engineering, London, Vol. 3, 30 June-2 July 2010, pp. 19231930.

http://www.iaeng.org/publication/WCE2010/WCE2010_ pp1923-1930.pdf

[8] A. Suryanto, "Stability and Bifurcation of a Discrete SIS Epidemic Model with a Delay," Proceedings of the 2nd International Conference on Basic Sciences, Malang, 24-25 February 2012, pp. 1-6.

[9] R. E. Mickens, "Application of Nonstandard Finite Difference Schemes," World Scientific Publishing Co Pte. Ltd., Singapore City, 2000. doi:10.1142/9789812813251

[10] R. E. Mickens, "Numerical Integration of Population Models Satisfying Conservation Laws: NSFD Methods," Journal of Biological Dynamics, Vol. 1, No. 4, 2007, pp. 427-436. doi:10.1080/17513750701605598

[11] D. T. Dimitrov and H. V. Kojouharov, "Nonstandard Finite Difference Method for Predator-Prey Models with General Functional Response," Mathematics and Computers in Simulation, Vol. 78, No. 1, 2008, pp. 1-11. doi:10.1016/j.matcom.2007.05.001

[12] A. J. Arenas, J. A. Morano and J. C. Cortés, "Non-Standard Numerical Method for a Mathematical Model of RSV Epidemiological Transmission," Computers and Mathematics with Applications, Vol. 56, No. 3, 2008, pp. 670-678. doi:10.1016/j.camwa.2008.01.010

[13] L. Jódar, R. J. Villanueva, A. J. Arenas and G. C. González, "Nonstandard Numerical Methods for a Mathematical Model for Influenza Disease," Mathematics and Computers in Simulation, Vol. 79, No. 3, 2008, pp. 622633. doi:10.1016/j.matcom.2008.04.008

[14] A. J. Arenas, G. González-Parra and B. M. Chen-Charpentier, "A Nonstandard Numerical Scheme of Predictor-Corrector Type for Epidemic Models," Computers and Mathematics with Applications, Vol. 59, No. 12, 2010, pp. 3740-3749. doi:10.1016/j.camwa.2010.04.006

[15] A. Suryanto, "A Dynamically Consistent Nonstandard Numerical Scheme for Epidemic Model with Saturated Incidence Rate," International Journal of Mathematics and Computation, Vol. 13, No. D11, 2011, pp. 112-123. http://ceser.in/ceserp/index.php/ijmc/article/view/1151

[16] A. Suryanto, “A Dynamically Consistent Numerical Method for SIRS Epidemic Model with Non-Monotone Incidence Rate," Proceedings of the 7th International Conference on Mathematics, Statistics and Its Application, Bangkok, 7-10 December 2011, pp. 273-282.

[17] C. Zhang, Y. Zu and B. Zheng, "Stability and Bifurcation of a Discrete Red Blood Cell Survival Model," Chaos, Solitons and Fractal, Vol. 28, No. 2, 2006, pp. 386-394. doi:10.1016/j.chaos.2005.05.042. 